Cahiers $d u$ MONDE RUSSE

\section{Cahiers du monde russe}

Russie - Empire russe - Union soviétique et États indépendants

$56 / 4 \mid 2015$

Médiateurs d'empire en Asie centrale (1820-1928)

\title{
Marina Mogilner, Homo Imperii, A History of Physical Anthropology in Russia
}

\section{Alain Blum}

\section{OpenEdition}

\section{Journals}

Édition électronique

URL : http://journals.openedition.org/monderusse/8243

DOI : 10.4000/monderusse.8243

ISSN : $1777-5388$

\section{Éditeur}

Éditions de l'EHESS

\section{Édition imprimée}

Date de publication : 1 octobre 2015

Pagination : 820-824

ISBN : 978-2-7132-2507-9

ISSN : $1252-6576$

Référence électronique

Alain Blum, "Marina Mogilner, Homo Imperii, A History of Physical Anthropology in Russia », Cahiers du monde russe [En ligne], 56/4 | 2015, mis en ligne le 01 octobre 2015, Consulté le 25 septembre 2020. URL : http://journals.openedition.org/monderusse/8243 ; DOI : https://doi.org/10.4000/ monderusse. 8243

Ce document a été généré automatiquement le 25 septembre 2020. 


\title{
Marina Mogilner, Homo Imperii, A History of Physical Anthropology in Russia
}

\author{
Alain Blum
}

\section{RÉFÉRENCE}

Marina MOGILNER, Homo Imperii, A History of Physical Anthropology in Russia,

Lincoln : University of Nebraska Press Critical Studies in the History of Anthropology, 2013, 486 p.

1 Homo Imperii est la traduction révisée bienvenue de l'ouvrage paru en russe en 2008 (Homo imperii : Istorija fizičeskoj antropologii v Rossii [Homo Imperii : Une histoire de l'anthropologie physique en Russie], M. : Novoe literaturnoe obozrenie, 2008). Marina Mogilner, l'une des éditrices de la revue $A b$ Imperio, présente une analyse tout à fait novatrice et passionnante de l'histoire de l'anthropologie physique dans l'Empire russe. Elle offre désormais un contrepoint indispensable aux très nombreux travaux portant sur l'anthropologie physique au $\mathrm{XIX}^{\mathrm{e}}$ siècle dans les autres pays européens, cette anthropologie dont l'ancrage dans une référence coloniale et raciale, impliquant hiérarchie et domination, est bien établi.

2 Son étude s'étend de la seconde moitié du XIX siècle à la Première Guerre mondiale, un chapitre conclusif s'interrogeant sur héritage, continuité et rupture de la période soviétique avec la période impériale.

3 Marina Mogilner offre un regard complexe sur cette anthropologie de l'Empire russe, qu'elle présente schématiquement comme fondée sur une orientation principale, une école dominante, libérale et que caractérise essentiellement la chaire d'anthropologie de l'université de Moscou. Mais cette dominante ne signifie pas exclusivité, et cette anthropologie libérale s'oppose à une orientation plus classique, traditionnelle en 
Europe, d'une anthropologie raciale, d'un "racisme académique ", développé en particulier à l'université Saint Vladimir de Kiev.

Elle étudie d'abord les racines des études académiques d'anthropologie physique dans l'Empire, en particulier au sein de l'association des amateurs des sciences naturelles, de l'anthropologie et de l'ethnographie (Imperatorskoe obščstvo ljubitelej estestvoznanija, antropologii i ètnografii - IOLEAĖ). Elle montre ensuite l'institutionnalisation de cette discipline quand, sous l'impulsion d'Aleksej Ivanovskij et surtout de Dmitrij Anučin à Moscou, se développe ce que Marina Mogilner nomme "l'anthropologie libérale ", rompant avec une anthropologie raciale hiérarchisante. Il s'agit d'un projet intégrateur, qui cherche à penser l'Empire russe comme un tout, disposant d'une grande variété de types anthropologiques, mais qui peuvent se décliner sous forme transversale. Il s'agit aussi d'un projet dans lequel, chez certains, en particulier Anučin, la relation entre culture et race est rompue.

5 L'anthropologie physique profite de l'immense espace de l'Empire et peut développer ses théories sans sortir de ce territoire. Les dimensions impériale et coloniale ont donc un effet direct sur les pratiques, effet structurel. Cependant l'anthropologie libérale s'oppose à la nationalisation du discours politique, se refusant à révéler un type physique russe. Dans une perspective universaliste, elle met en évidence en tout lieu la multiplicité des types, la mixité des populations. "All possible tensions were sublimated in the concept of the mixed racial type that became the mantra of the Russian liberal anthropology of imperial diversity » (p. 108).

Ce projet montre cependant ses limites lorsqu'il est question des juifs. Cette science russe libérale de la race ne peut aller jusqu'au bout de la logique intégratrice. Les juifs apparaissent comme les "universal others ", indispensable point négatif du projet intégrationniste ; la "physiognomie juive ", cette science des apparences physiques si présente au $\mathrm{XIX}^{\mathrm{e}}$ siècle, permet d'introduire cette distance ambivalente, posant les limites de l'approche anthropologique défendue par Anučin.

7 Cette anthropologie libérale, qui a manifestement les faveurs de Marina Mogilner, s'oppose à l'anthropologie raciale d'Ivan Sikorskij, plus ancrée dans la vision coloniale et raciale très présente en Europe au même moment. À la différence de l'anthropologie libérale, cette vision alternative n'hésite pas à user du terme "russes » comme décrivant un type particulier, national. Sikorskij mêle un discours fortement nationaliste et des analyses anthropologiques.

D'un point de vue politique plus général, Marina Mogilner montre la relative indifférence de l'État impérial russe vis-à-vis de l'anthropologie, qu'elle soit libérale ou nationaliste. Les autorités impériales laissent ceux qui s'en emparent user des méthodes qu'ils veulent sans pour autant les encourager. En revanche, elles portent un intérêt particulier à cette question dès qu'il s'agit de la gestion de la diversité dans l'armée. Une partie de l'ouvrage est consacrée à l'anthropologie militaire qui se développe à partir des réformes des années 1860 et 1870 . Elle met en évidence la réflexion menée par les anthropologues militaires, portant sur l'usage militaire des divers types physiques. Ils s'interrogent en particulier sur leur plus ou moins grande «adaptation » au service. Cela conduit progressivement les anthropologues militaires à penser le contingent en termes d'efficacité maximale, par exclusion des types raciaux inadéquats, assumant de plus en plus une discrimination raciale, à partir des années 1910, qui éloigne de la vision d'une «armée impériale » inclusive pour aller vers une « armée nationale ». 
9 Une autre dimension passionnante de cet ouvrage est l'analyse, qui court tout au long du livre sans devenir l'objet d'un chapitre spécifique, des formes de scientificité, des mécanismes de démonstration, qui conduisent aux sciences des types physiques dont on connaît l'issue fatale. Quelle preuve pouvait donc être avancée, alors que ces démarches ont perdu, aujourd'hui, toute scientificité. Mogilner nous offre à plusieurs reprises de très belles pages portant sur ces questions. Son chapitre sur l'analyse des cerveaux humains en est le plus bel exemple, s'inscrivant dans une longue tradition d'histoire des sciences se penchant sur ces questions. Elle analyse ainsi en détail les travaux de Nikolaj Gil'čenko en portant une attention particulière aux «démonstrations » proposées par cet auteur. D’un côté, Gil'čenko mène ses recherches dans la plus pure tradition coloniale, disposant à volonté des cerveaux des soldats décédés de tous les peuples de l'Empire, sans qu'il ait reçu de quelconque accord ni qu'il ait tenu compte des pratiques culturelles. Il constitue ainsi une immense collection de mesures, dans la plus pure tradition coloniale de l'anthropologie physique. D'un autre côté, il développe des approches qui le conduisent à penser l'Empire comme un tout et à nier des hiérarchies raciales fondées sur ces mesures. "The rich data obtained by Gil 'chenko, on the contrary [d'utiliser ces mesures comme marqueurs quantitatifs de races «supérieures" ou "dégénérées »], displayed a considerable disparity of indicators of weight and simply disporve the colonial logic embedded in the study of "fresh brain" " (p. 263). Autre "démontage " d'une démonstration scientifique, les pages écrites par Marina Mogilner sur les analyses d'anthropologie physique qu'Anučin d'un côté, Sikorskij de l'autre, développent sur Puškin, sont un exemple du genre. Elle montre d'un côté comment, dans ce cas, Anučin s'éloigne de ses thèses libérales pour introduire une relation entre race et culture et démontrer que Puškin est bien européen, tout en assumant sa mixité raciale. Il est contraint de séparer la «race éthiopienne " de la "race nègre ", pour arriver à son but. De son côté Sikorskij, enfermé dans un cadre nationaliste qui lui est propre, ne s'attache pas à résoudre la question de la mixité, mais construit son modèle sur l'idée d'absorption d'une race par une autre, et donc de la domination des origines russes chez Puškin.

Cet ouvrage, fondé sur un matériau d'une très grande richesse, jusqu'à présent très mal connu, est sans aucun doute le premier qui aborde avec une telle ampleur l'histoire de l'anthropologie physique en Russie. Il mêle une histoire des milieux académiques, militaires, politiques (dans une moindre mesure cependant), une histoire de la circulation des idées entre la France, l'Italie, l'Allemagne et l'Empire russe ; une histoire aussi des lieux de constitution de ces pratiques dans l'Empire et de leur impact sur son développement contradictoire. Il est aussi exemplaire comme histoire des pratiques de démonstration, du développement de la preuve dans une science dont on sait aujourd'hui qu'elle fut une impasse et dont on comprend l'inanité de l'immense appareil d'observation et de démonstration qu'il a mis en œuvre. C'est une contribution essentielle à l'histoire des sciences européennes, à l'histoire des sciences dans un Empire, dans les perspectives intégratrices ou nationalistes. Il s'inscrit aussi désormais entièrement dans tout un ensemble de recherches qui ont porté sur l'usage des sciences dans la représentation puis la gestion des nationalités dans l'Empire et en URSS².

11 Notons pour conclure l'intéressante polémique qu'il a contribué à alimenter, ouverte dans la revue Laboratorium, entre Aleksandr Etkind ${ }^{3}$, d'une part, Vera Tolz et Konstantin Ivanov $^{4}$ d'autre part, à partir de la version russe de l'ouvrage. Le premier, tout en faisant un commentaire tout à fait laudateur au travail de Marina Mogilner, lui 
reproche de ne pas tenir compte de l'impasse dans laquelle est tombée l'anthropologie physique et surtout de son usage au $\mathrm{xx}^{\mathrm{e}}$ siècle. Pour Vera Tolz, une histoire des sciences ne peut avoir de perspective téléologique, alors que pour Konstantin Ivanov, ce ne fut pas qu'une impasse puisque l'anthropologie physique a offert des outils à la biologie de l'évolution. Il souligne aussi d'autres aspects importants de l'usage de ces travaux. Sans doute Aleksandr Etkind reproche-t-il implicitement une empathie de Marina Mogilner pour ses " héros » d'une anthropologie libérale, mais il n'en est pas moins vrai qu'en offrant une analyse des démonstrations, elle témoigne d'une perspective qui n'omet pas le futur de cette discipline et ses conséquences, même si elle ne le fait jamais intervenir explicitement. Son interprétation en termes d'universalisme ou science de la diversité d'un Empire versus nationalisme est à l'évidence influencée par le devenir et les conséquences de cette science. Il est vrai que Marina Mogilner, pour ne pas s'inscrire dans une histoire des sciences téléologique, ne pose pas cette forme d'influence sur ses propres interprétations. On sera, il est vrai, d'accord avec Etkind pour ne pas se cantonner dans une approche qui, par précaution méthodologique et pour éviter tout anachronisme ou téléologie, exclurait entièrement le futur de cette discipline comme éclairant son historicité. Il serait illusoire (et non pertinent) de penser que notre analyse puisse faire abstraction d'un regard contemporain sur ces questions. Mais, l'inscription dans le contexte de la fin du XIXe siècle est tout aussi essentielle, ce que fait parfaitement Marina Mogilner.

\section{NOTES}

1. » Les riches données obtenues par Gil'čenko, au contraire [d'utiliser ces mesures comme marqueurs quantitatifs de races "supérieures » ou " dégénérées »], offrent des indicateurs de poids d'une considérable disparité et réfutent simplement la logique coloniale présente dans l'étude des « cerveaux frais ».

2. En particulier, Juliette Cadiot, Le laboratoire impérial Russie-URSS, 1870-1940, P. : CNRS éd., 2007 ; Francine Hirsch, Empire of Nations : Ethnographic Knowledge \& the Making of the Soviet Union, Ithaca : Cornell University Press, 2005 ; Peter Holquist, " To Count, to Extract, to Exterminate : Population Statistics and Population Politics in Late Imperial and Soviet Russia ", in Ronald Grigor Suny et Terry Dean Martin, dir., A State of Nations : Empire and Nation-Making in the Age of Lenin and Stalin, Oxford : Oxford University Press, 2001, vol. 1.

3. Alexander Etkind, « Marina Mogil'ner. Homo imperii. Istoriia fizičeskoi antropologij v Rossii [Marina Mogil'ner. Homo imperii. Histoire de l'anthropologie physique en Russie] », M. : NLO, 2008, Laboratorium : Russian Review of Social Research, 3 (2), 1 octobre 2011, p. 90-93.

4. Vera Tolz, « Response to Alexander Etkind's review of Marina Mogilner's Homo imperii : Istoriia fizičeskoj antropologii v Rossii ", Laboratorium : Russian Review of Social Research, 3 (2), 1 octobre 2011, p. $94-96$; Konstantin Ivanov, « Replika po povodu diskussii o knige Mariny Mogil 'ner "Homo imperii : Istoriia fizičeskoj antropologii v Rossii" ", Laboratorium : Russian Review of Social Research, 3 (2), 1 octobre 2011, p. 97-99. 


\section{AUTEURS}

ALAIN BLUM

Ehess - Ined 